\title{
Acta Veterinaria Scandinavica reviewer acknowledgement 2013
}

Jørgen S Agerholm

\section{Contributing reviewers}

The Acta Veterinaria Scandinavica editorial team would like to thank the following colleagues who contributed to peer review for the journal in 2013.

\section{Luis Calvinho}

Argentina

David Evans

Australia

Frank Nicholas

Australia

Nerissa Stander

Australia

Michael J Studdert

Australia

Martina Flöck

Austria

Michaela Gumpenberger

Austria

Ernst Leidinger

Austria

Christoph Winckler

Austria

Ahmad Rabiee

Azerbaijan

Christian Burvenich

Belgium

Undine Christmann

Belgium
Jeroen Dewulf

Belgium

Maarten Hoogewijs

Belgium

Dominiek Maes

Belgium

Davy Persoons

Belgium

Bart Van Goethem

Belgium

Maira Souza Oliveira

Brazil

Carlos Termignoni

Brazil

Sebastien Buczinski

Canada

Etienne Côté

Canada

José Denis-Robichaud

Canada

\section{A Hossain Farid \\ Canada}

Martin Guillot

Canada
Janet Hill

Canada

Stephen LeBlanc

Canada

Kathleen Linn

Canada

Christopher Luby

Canada

Jérôme Planté

Canada

Scott Weese

Canada

Klas Abelson

Denmark

Jens Agger

Denmark

\section{Aage Alstrup}

Denmark

\section{Mads Bertelsen}

Denmark

Peter Bollen

Denmark

Rikke Buhl

Denmark

Correspondence: jager@sund.ku.dk

Department of Large Animal Sciences, Faculty of Health and Medical

Sciences, University of Copenhagen, Dyrlægevej 68, DK-1870 Frederiksberg C,

Denmark

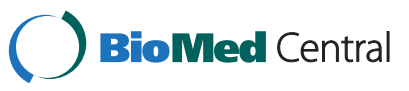

(c) 2014 Agerholm; licensee BioMed Central Ltd. This is an Open Access article distributed under the terms of the Creative Commons Attribution License (http://creativecommons.org/licenses/by/4.0), which permits unrestricted use, distribution, and reproduction in any medium, provided the original work is properly credited. The Creative Commons Public Domain Dedication waiver (http://creativecommons.org/publicdomain/zero/1.0/) applies to the data made available in this article, unless otherwise stated. 
Henrik Callesen

Denmark

Henrik Christensen

Denmark

Charlotte Maria Dalsgaard

Denmark

Birthe Damgaard

Denmark

Jörg Matthias Dehn Enemark

Denmark

Rikke Fast

Denmark

Anne Sofie Hammer

Denmark

Anne-Marie Heegaard

Denmark

Mette Herskin

Denmark

Stine Jacobsen

Denmark

Henrik Elvang Jensen

Denmark

Anders Ringgaard Kristensen

Denmark

Anders Larsen

Denmark

Nanna Luthersson

Denmark

Jens Malmkvist

Denmark

Line Morgills

Denmark

Ole Lerberg Nielsen

Denmark

\section{Lisbeth Høier Olsen}

Denmark

Karl Pedersen

Denmark

Mette Schmidt

Denmark

Paul E Simonsen

Denmark

Mette Tingleff Skaanild

Denmark
Tine Søland

Denmark

Dorte Bratbo Sørensen

Denmark

Pernille Tveden-Nyborg

Denmark

Håkan Vigre

Denmark

Brian Lassen

Estonia

Toomas Orro

Estonia

Arvo Viltrop

Estonia

Andres Waldmann

Estonia

Magnus Carl Andersson

Finland

Marjukka Anttila

Finland

Laura Hänninen

Finland

Marja-Liisa Hänninen

Finland

Mari Heinonen

Finland

Udo Hetzel

Finland

Anita Huovilainen

Finland

Liisa Kaartinen

Finland

Paula M Kinnunen

Finland

Outi Laitinen-Vapaavuori

Finland

Anna-Liisa Myllyniemi

Finland

Mauri Nieminen

Finland

Raimo Pohjanvirta

Finland

Satu Pyörälä

Finland
Merja Rantala

Finland

Helena Rautala

Finland

Liisa Helena Sihvonen

Finland

Juhani Taponen

Finland

Suvi Taponen

Finland

Riitta-Mari Tulamo

Finland

Pirkko Lucia Tuominen

Finland

Pekka Uimari

Finland

François Casas

France

Gilles Charpigny

France

Xavier Druart

France

Gilles Foucras

France

Lucia Manso-Silvan

France

Emmanuelle Robardet

France

Sally Solomon

France

Francois Thiaucourt

France

Berit Bangoura

Germany

Armando Damiani

Germany

Viktor Dyachenko

Germany

Manfred Kietzmann

Germany

Wolfgang Klee

Germany

Stefanie Klenner

Germany 


\begin{tabular}{|c|c|c|}
\hline $\begin{array}{l}\text { Robert Klopfleisch } \\
\text { Germany }\end{array}$ & $\begin{array}{l}\text { Nicola Decaro } \\
\text { Italy }\end{array}$ & $\begin{array}{l}\text { Terje Fjeldaas } \\
\text { Norway }\end{array}$ \\
\hline Klaus Henning & Luigi Calamari & Tore Framstad \\
\hline Germany & Italy & Norway \\
\hline $\begin{array}{l}\text { Wolfgang Holtz } \\
\text { Germany }\end{array}$ & $\begin{array}{l}\text { Angela Di Cesare } \\
\text { Italy }\end{array}$ & $\begin{array}{l}\text { Anna Germundsson } \\
\text { Norway }\end{array}$ \\
\hline $\begin{array}{l}\text { Arno Lindner } \\
\text { Germany }\end{array}$ & $\begin{array}{l}\text { Gaetano Mari } \\
\text { Italy }\end{array}$ & $\begin{array}{l}\text { Karin Hultin Jäderlund } \\
\text { Norway }\end{array}$ \\
\hline $\begin{array}{l}\text { Burkhard Malorny } \\
\text { Germany }\end{array}$ & $\begin{array}{l}\text { Paolo Pasquali } \\
\text { Italy }\end{array}$ & $\begin{array}{l}\text { Carl Fredrik Ihler } \\
\text { Norway }\end{array}$ \\
\hline $\begin{array}{l}\text { Reinhard Mischke } \\
\text { Germany }\end{array}$ & $\begin{array}{l}\text { Alessandro Poli } \\
\text { Italy }\end{array}$ & $\begin{array}{l}\text { Paal Johnsen } \\
\text { Norway }\end{array}$ \\
\hline $\begin{array}{l}\text { Karin Müller } \\
\text { Germany }\end{array}$ & $\begin{array}{l}\text { Monica Probo } \\
\text { Italy }\end{array}$ & $\begin{array}{l}\text { Hannah Joan Jørgensen } \\
\text { Norway }\end{array}$ \\
\hline $\begin{array}{l}\text { Ralf Müller } \\
\text { Germany }\end{array}$ & $\begin{array}{l}\text { Tetsuo Asai } \\
\text { Japan }\end{array}$ & $\begin{array}{l}\text { Randi Ingebjørg Krontveit } \\
\text { Norway }\end{array}$ \\
\hline $\begin{array}{l}\text { Heinrich Neubauer } \\
\text { Germany }\end{array}$ & $\begin{array}{l}\text { Junko Kohara } \\
\text { Japan }\end{array}$ & $\begin{array}{l}\text { Thor Landsverk } \\
\text { Norway }\end{array}$ \\
\hline $\begin{array}{l}\text { Gerald Rimbach } \\
\text { Germany }\end{array}$ & $\begin{array}{l}\text { Makoto Miyata } \\
\text { Japan }\end{array}$ & $\begin{array}{l}\text { Bjørn Lium } \\
\text { Norway }\end{array}$ \\
\hline $\begin{array}{l}\text { Hans-Joachim Schuberth } \\
\text { Germany }\end{array}$ & $\begin{array}{l}\text { Susanne Boroffka } \\
\text { Netherlands }\end{array}$ & $\begin{array}{l}\text { Tormod Mork } \\
\text { Norway }\end{array}$ \\
\hline $\begin{array}{l}\text { Gabriele Stangl } \\
\text { Germany }\end{array}$ & $\begin{array}{l}\text { Menno Holzhauer } \\
\text { Netherlands }\end{array}$ & $\begin{array}{l}\text { Stephen Mutoloki } \\
\text { Norway }\end{array}$ \\
\hline $\begin{array}{l}\text { Michael Wendt } \\
\text { Germany }\end{array}$ & $\begin{array}{l}\text { Paul Mandigers } \\
\text { Netherlands }\end{array}$ & $\begin{array}{l}\text { Liv Torunn Mydland } \\
\text { Norway }\end{array}$ \\
\hline $\begin{array}{l}\text { Wilhelm Windisch } \\
\text { Germany }\end{array}$ & $\begin{array}{l}\text { Auke Schaefers-Okkens } \\
\text { Netherlands }\end{array}$ & $\begin{array}{l}\text { Anne Berit Olsen } \\
\text { Norway }\end{array}$ \\
\hline $\begin{array}{l}\text { Ilias Giannenas } \\
\text { Greece }\end{array}$ & $\begin{array}{l}\text { Viktor Szatmári } \\
\text { Netherlands }\end{array}$ & $\begin{array}{l}\text { Liv Jorun Reitan } \\
\text { Norway }\end{array}$ \\
\hline $\begin{array}{l}\text { Tomas Guerrero } \\
\text { Grenada }\end{array}$ & $\begin{array}{l}\text { Cornelis van Elk } \\
\text { Netherlands }\end{array}$ & $\begin{array}{l}\text { Bente Kristin Sævik } \\
\text { Norway }\end{array}$ \\
\hline $\begin{array}{l}\text { Szabolcs Nagy } \\
\text { Hungary }\end{array}$ & $\begin{array}{l}\text { Frederik J van Sluijs } \\
\text { Netherlands }\end{array}$ & $\begin{array}{l}\text { Tore Sivertsen } \\
\text { Norway }\end{array}$ \\
\hline $\begin{array}{l}\text { Grétar Harơarson } \\
\text { Iceland }\end{array}$ & $\begin{array}{l}\text { Jon Hickford } \\
\text { New Zealand }\end{array}$ & $\begin{array}{l}\text { Ellen Skancke } \\
\text { Norway }\end{array}$ \\
\hline $\begin{array}{l}\text { Lena Englund } \\
\text { Ireland }\end{array}$ & $\begin{array}{l}\text { Keith Thompson } \\
\text { New Zealand }\end{array}$ & $\begin{array}{l}\text { Eystein Skjerve } \\
\text { Norway }\end{array}$ \\
\hline $\begin{array}{l}\text { Itamar Aroch } \\
\text { Israel }\end{array}$ & $\begin{array}{l}\text { Jon M Arnemo } \\
\text { Norway }\end{array}$ & $\begin{array}{l}\text { Hege Kippenes Skogmo } \\
\text { Norway }\end{array}$ \\
\hline $\begin{array}{l}\text { Mark Pines } \\
\text { Israel }\end{array}$ & $\begin{array}{l}\text { Nils Ivar Dolvik } \\
\text { Norway }\end{array}$ & $\begin{array}{l}\text { Snorre Stuen } \\
\text { Norway }\end{array}$ \\
\hline $\begin{array}{l}\text { Tiziana Bellini } \\
\text { Italy }\end{array}$ & $\begin{array}{l}\text { Arild Espenes } \\
\text { Norway }\end{array}$ & $\begin{array}{l}\text { Synnøve Vatn } \\
\text { Norway }\end{array}$ \\
\hline
\end{tabular}




\begin{tabular}{|c|c|c|}
\hline $\begin{array}{l}\text { Arne Yndestad } \\
\text { Norway }\end{array}$ & $\begin{array}{l}\text { Kerstin Bergvall } \\
\text { Sweden }\end{array}$ & $\begin{array}{l}\text { Manja Zupan } \\
\text { Sweden }\end{array}$ \\
\hline $\begin{array}{l}\text { Malgorzata Brzóska } \\
\text { Poland }\end{array}$ & $\begin{array}{l}\text { Orjan Carlborg } \\
\text { Sweden }\end{array}$ & $\begin{array}{l}\text { Valentino Cattori } \\
\text { Switzerland }\end{array}$ \\
\hline $\begin{array}{l}\text { Tadeusz Frymus } \\
\text { Poland }\end{array}$ & $\begin{array}{l}\text { Agneta Egenvall } \\
\text { Sweden }\end{array}$ & $\begin{array}{l}\text { Mark Flückiger } \\
\text { Switzerland }\end{array}$ \\
\hline $\begin{array}{l}\text { Jacek Gajek } \\
\text { Poland }\end{array}$ & $\begin{array}{l}\text { Stig Einarsson } \\
\text { Sweden }\end{array}$ & $\begin{array}{l}\text { Paola Gandolfi-De } \\
\text { Switzerland }\end{array}$ \\
\hline $\begin{array}{l}\text { Urszula Paslawska } \\
\text { Poland }\end{array}$ & $\begin{array}{l}\text { Stina Ekman } \\
\text { Sweden }\end{array}$ & $\begin{array}{l}\text { David Spreng } \\
\text { Switzerland }\end{array}$ \\
\hline $\begin{array}{l}\text { Agata Piestrzynska-Kajtoch } \\
\text { Poland }\end{array}$ & $\begin{array}{l}\text { Marianne Elvander } \\
\text { Sweden }\end{array}$ & $\begin{array}{l}\text { Adrian Steiner } \\
\text { Switzerland }\end{array}$ \\
\hline $\begin{array}{l}\text { Piotr Szefer } \\
\text { Poland }\end{array}$ & $\begin{array}{l}\text { Helle Ericsson Unnerstad } \\
\text { Sweden }\end{array}$ & $\begin{array}{l}\text { Monika Welle } \\
\text { Switzerland }\end{array}$ \\
\hline $\begin{array}{l}\text { Dariusz Wasyl } \\
\text { Poland }\end{array}$ & $\begin{array}{l}\text { Ingrid Hansson } \\
\text { Sweden }\end{array}$ & $\begin{array}{l}\text { Mark Bowen } \\
\text { United Kingdom }\end{array}$ \\
\hline $\begin{array}{l}\text { Tomasz Wielkoszynski } \\
\text { PolandPoland }\end{array}$ & $\begin{array}{l}\text { Eva Hellmén } \\
\text { Sweden }\end{array}$ & $\begin{array}{l}\text { Marnie Brennan } \\
\text { United Kingdom }\end{array}$ \\
\hline $\begin{array}{l}\text { Fatima Gartner } \\
\text { Portugal }\end{array}$ & $\begin{array}{l}\text { Odd Viking Hoglund } \\
\text { Sweden }\end{array}$ & $\begin{array}{l}\text { Lucy Brunton } \\
\text { United Kingdom }\end{array}$ \\
\hline $\begin{array}{l}\text { Peter Massanyi } \\
\text { Slovakia }\end{array}$ & $\begin{array}{l}\text { Kjell Holtenius } \\
\text { Sweden }\end{array}$ & $\begin{array}{l}\text { Harry Carslake } \\
\text { United Kingdom }\end{array}$ \\
\hline $\begin{array}{l}\text { Dusan Bencina } \\
\text { Slovenia }\end{array}$ & $\begin{array}{l}\text { Magdalena Jacobson } \\
\text { Sweden }\end{array}$ & $\begin{array}{l}\text { Fiona Cooke } \\
\text { United Kingdom }\end{array}$ \\
\hline $\begin{array}{l}\text { Marjeta Candek-Potokar } \\
\text { Slovenia }\end{array}$ & $\begin{array}{l}\text { Anna Jansson } \\
\text { Sweden }\end{array}$ & $\begin{array}{l}\text { Robert Davies } \\
\text { United Kingdom }\end{array}$ \\
\hline $\begin{array}{l}\text { Martie van der Walt } \\
\text { South Africa }\end{array}$ & $\begin{array}{l}\text { Hans Kindahl } \\
\text { Sweden }\end{array}$ & $\begin{array}{l}\text { Steven De Decker } \\
\text { United Kingdom }\end{array}$ \\
\hline $\begin{array}{l}\text { Sonia Almeria } \\
\text { Spain }\end{array}$ & $\begin{array}{l}\text { Leif Kirsebom } \\
\text { Sweden }\end{array}$ & $\begin{array}{l}\text { Hilary Dobson } \\
\text { United Kingdom }\end{array}$ \\
\hline $\begin{array}{l}\text { Librado Carrasco } \\
\text { Spain }\end{array}$ & $\begin{array}{l}\text { Anne-Sofie Lagerstedt } \\
\text { Sweden }\end{array}$ & $\begin{array}{l}\text { Laura Green } \\
\text { United Kingdom }\end{array}$ \\
\hline $\begin{array}{l}\text { Pia Haubro Andersen } \\
\text { Sweden }\end{array}$ & $\begin{array}{l}\text { Charles Ley } \\
\text { Sweden }\end{array}$ & $\begin{array}{l}\text { Edward Hall } \\
\text { United Kingdom }\end{array}$ \\
\hline $\begin{array}{l}\text { Eva Axnér } \\
\text { Sweden }\end{array}$ & $\begin{array}{l}\text { Cecilia Rohdin } \\
\text { Sweden }\end{array}$ & $\begin{array}{l}\text { Katherine Hughes } \\
\text { United Kingdom }\end{array}$ \\
\hline $\begin{array}{l}\text { Renée Båge } \\
\text { Sweden }\end{array}$ & $\begin{array}{l}\text { Bengt Röken } \\
\text { Sweden }\end{array}$ & $\begin{array}{l}\text { Jolyon Medlock } \\
\text { United Kingdom }\end{array}$ \\
\hline $\begin{array}{l}\text { Viveca Båverud } \\
\text { Sweden }\end{array}$ & $\begin{array}{l}\text { Bodil Strom Holst } \\
\text { Sweden }\end{array}$ & $\begin{array}{l}\text { Susan Paterson } \\
\text { United Kingdom }\end{array}$ \\
\hline $\begin{array}{l}\text { Bjorn Bengtsson } \\
\text { Sweden }\end{array}$ & $\begin{array}{l}\text { Anna Tidholm } \\
\text { Sweden }\end{array}$ & $\begin{array}{l}\text { Simon Priestnall } \\
\text { United Kingdom }\end{array}$ \\
\hline $\begin{array}{l}\text { Anna Bergh } \\
\text { Sweden }\end{array}$ & $\begin{array}{l}\text { Per Wallgren } \\
\text { Sweden }\end{array}$ & $\begin{array}{l}\text { Tobias Schwarz } \\
\text { United Kingdom }\end{array}$ \\
\hline
\end{tabular}


Phil Scott

United Kingdom

Jenny Stavisky

United Kingdom

Frank Andrews

United States of America

Paul Bartlett

United States of America

\section{Alvin Camus}

United States of America

Thomas Casey

United States of America

Roger Clemmons

United States of America

Hans Coetzee

United States of America

James Evermann

United States of America

John C Godbold Jr

United States of America

Barbara Gores

United States of America

John Griffin

United States of America

Rita Hanel

United States of America

Steve Hodges

United States of America
Michele Jay-Russell

United States of America

Mark Jenkins

United States of America

Anil Kadegowda

United States of America

Katherine Kocan

United States of America

Bradley Kropp

United States of America

Michelle Kutzler

United States of America

Paulraj Lawrence

United States of America

Jane Manfredi

United States of America

Stanley Marks

United States of America

Mark Mitchell

United States of America

Tiruvoor Nagaraja

United States of America

Stephen Nickerson

United States of America

Annette O'Connor

United States of America

Robert Paine

United States of America
Elena Alina Palade

United States of America

Mary Pantin-Jackwood

United States of America

Narda Robinson

United States of America

Subramaniam Srikumaran

United States of America

Olivier Taeymans

United States of America

\section{John Timoney}

United States of America

Betsy Vaughan

United States of America

Deborah Vela

United States of America

Robert Washabau

United States of America

Robert Weedon

United States of America

Zheng Xing

United States of America

Laszlo Zsak

United States of America

Alejo Menchaca

Uruguay

Scandinavica 2014 56:15. 